\title{
Formation and function of the manchette and flagellum during spermatogenesis
}

\author{
M S Lehti ${ }^{1,2}$ and A Sironen ${ }^{1}$ \\ ${ }^{1}$ Natural Resources Institute Finland (Luke), Green Technology, Fl-31600 Jokioinen, Finland and ${ }^{2}$ Department of \\ Physiology, Institute of Biomedicine, University of Turku, FI-20520 Turku, Finland
}

Correspondence should be addressed to A Sironen; Email: anu.sironen@luke.fi

\begin{abstract}
The last phase of spermatogenesis involves spermatid elongation (spermiogenesis), where the nucleus is remodeled by chromatin condensation, the excess cytoplasm is removed and the acrosome and sperm tail are formed. Protein transport during spermatid elongation is required for correct formation of the sperm tail and acrosome and shaping of the head. Two microtubular-based protein delivery platforms transport proteins to the developing head and tail: the manchette and the sperm tail axoneme. The manchette is a transient skirt-like structure surrounding the elongating spermatid head and is only present during spermatid elongation. In this review, we consider current understanding of the assembly, disassembly and function of the manchette and the roles of these processes in spermatid head shaping and sperm tail formation. Recent studies have shown that at least some of the structural proteins of the sperm tail are transported through the intra-manchette transport to the basal body at the base of the developing sperm tail and through the intraflagellar transport to the construction site in the flagellum. This review focuses on the microtubule-based mechanisms involved and the consequences of their disruption in spermatid elongation.
\end{abstract}

Reproduction (2016) 151 R43-R54

\section{Introduction}

Spermatogenesis is a complex process that begins with mitotic proliferation of spermatogonia, after which spermatocytes undergo meiotic divisions to give rise to haploid round spermatids. During the last phase of spermatogenesis, spermiogenesis, these haploid germ cells undergo substantial changes including condensation and elongation of the sperm head and development of the sperm tail. These morphological changes are crucial for the production of viable sperm and male fertility. In this review, we focus on the role of a transient microtubular platform in elongating spermatids, the manchette and its importance in the nuclear shaping and sperm tail formation. The manchette and core sperm tail structure, the axoneme, are microtubular platforms, which consist of $\alpha$ - and $\beta$-tubulin heterodimers; $\alpha$-tubulin is exposed at the minus-end and $\beta$-tubulin at the plus-end. The plus-end is able to grow and shrink rapidly, whereas the minus-end is more stable and anchored to the microtubuleorganizing center (MTOC) during nucleation. The current hypothesis is that proteins are transported through the manchette by intra-manchette transport (IMT) to the base of the sperm tail and to the developing sperm tail by intra-flagellar transport (IFT). Both of these transport mechanisms utilize microtubule tracks and motor proteins for translocation of multi-subunit cargo complexes. The mechanisms of IMT are not well known, but it has been suggested to resemble IFT. IFT is bidirectional movement along the axoneme using motor complexes of kinesin-2 for anterograde and cytoplasmic dynein-2 for retrograde movement. These motor proteins move IFT complex $B$ and $A$ respectively along the axoneme and enable cilia formation and function. Recently, IFT has been postulated also outside the cilium, particularly in targeted delivery of Golgiderived vesicles (Baldari \& Rosenbaum 2010).

\section{Manchette assembly and disassembly}

During spermatogenesis in the mouse, the manchette can be first seen in step 8 spermatids, when it rapidly forms a microtubular platform between the perinuclear ring surrounding the nucleus and the elongated sperm axoneme (O'Donnell \& O'Bryan 2014). The basic platform of the manchette consists of microtubules and actin filaments. Actin and the associated motor protein myosin play an important role in, e.g., vesicle transport and shaping the spermatid head due to their functional roles in the acroplaxome and manchette (Sun et al. 2011, Shen et al. 2014). Disassembly of the manchette occurs around steps 13-14 prior to the sperm tail mid-piece formation. The initiation of the manchette microtubule 
formation requires a nucleator. The emanation of the manchette microtubules is currently under debate. Two specific nucleation sites have been postulated for the manchette microtubules: the perinuclear ring and the centrosome.

\section{Evidence for the perinuclear ring as the nucleation site}

An investigation of microtubular dynamics during mammalian spermatogenesis suggested the appearance of microtubules first to the post-acrosomal region in mouse and bovine spermatids (Moreno \& Schatten 2000), where the periodic densities in the perinuclear ring might function as MTOC (Russell et al. 1991). The possibility that the perinuclear ring serves as the nucleation site is supported by observations that the free ends of the manchette are more dynamic than the perinuclear ring end (Yoshida et al. 1994). Furthermore, abnormal elongation of the manchette in many knockout (KO) mouse models implicates microtubular elongation at the caudal end of the manchette. Such observations are consistent with microtubules growing into the cytoplasm, with growth being regulated by the microtubule severing complex (O'Donnell et al. 2012). However, the nucleation of the microtubules at the perinuclear ring is not evident based on the current knowledge. The perinuclear ring lacks the well-known marker for MTOC, $\gamma$-tubulin. Thus, alternative nucleation sites should be considered.

\section{Evidence for the centrosome as the nucleation site}

The lack of $\gamma$-tubulin in the perinuclear ring implies that the centriolar adjunct also serves as the MTOC for manchette microtubules. Consistent with the localization of the nucleator $\gamma$-tubulin in the centrosomal adjunct, the microtubule plus-end tracking proteins CLIP-170, EB3 and dynactin components have been located near or at the perinuclear ring (Akhmanova et al. 2005, Kierszenbaum et al. 2011a, Fig. 1). The treatment with alkylating agent to inhibit the transcription during rat spermatogenesis resulted in a gap between the acrosome and the caudal manchette, and administration of microtubule stabilizing agent resulted in caudally located manchettes indenting into the nucleus (Russell et al. 1991). Interestingly, the nucleation of the manchette from the centrosome is also supported by an in vitro model, where exogenous taxol and GTP nucleate manchette microtubules with spermatid centrosomes (Kierszenbaum et al. 2002).

\section{Alternative hypotheses for the manchette formation}

Conflicting reports in the literature require further studies to confirm unequivocally the organization of manchette microtubules. It has also been suggested that

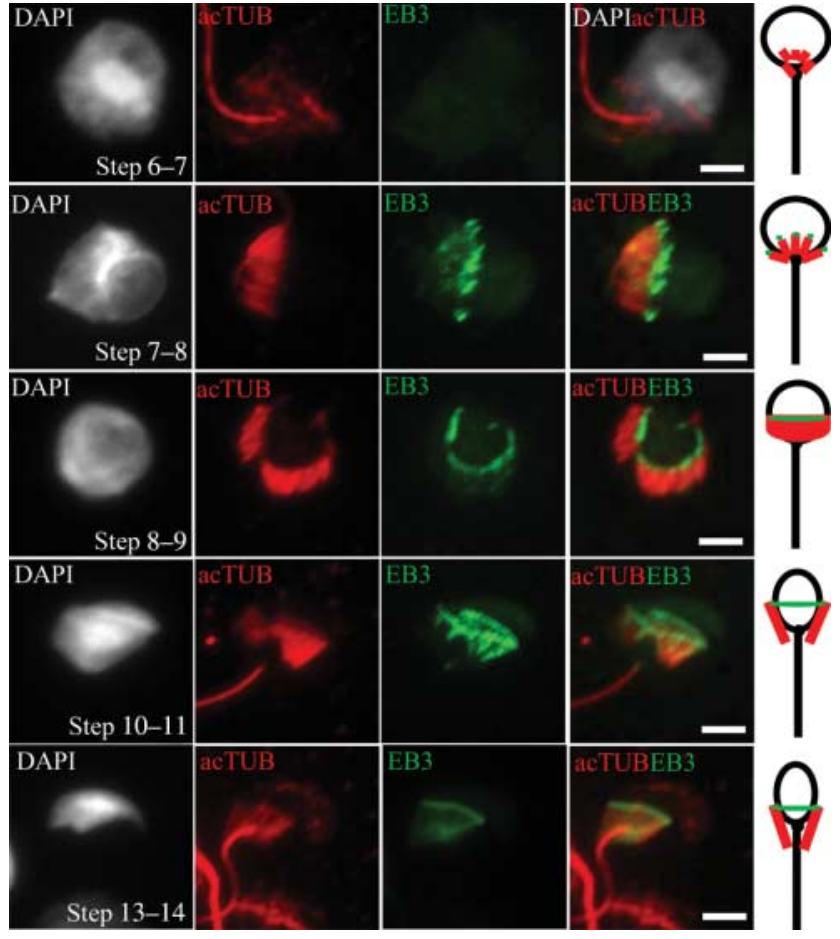

Figure 1 Manchette formation based on acetylated $\boldsymbol{\alpha}$-tubulin (acTUB) and microtubule plus-end tracking protein EB3 in the mouse.

Step-by-step investigation of WT mouse testicular cell spreads stained with acTUB and EB3 revealed a possible model for the manchette assembly. Microtubules (red) are detected near caudal end of the sperm head at spermiogenic steps 6-7. During steps 7-8, when manchette begins to develop, EB3 (green) appears at the plus-ends of the microtubules reaching toward the acrosomal end of the spermatid head. In steps 8-9 spermatids, EB3 localizes at the perinuclear ring and manchette microtubules appear to detach from the centrosomal region. EB3 staining is detected in the perinuclear ring until manchette removal at step 14. Scale bar $5 \mu \mathrm{m}$.

microtubules can be nucleated from existing microtubules (Ishihara et al. 2014), while other sites in the cytoplasm have also been implicated. Even a hypothesis that the manchette microtubules are nucleated prior to the pachytene spermatocyte stage and stored until required for manchette formation in elongating spermatids (Moreno \& Schatten 2000) has been introduced. However, these hypotheses have not been proven. Centrobin, which is required for microtubule nucleation and stabilization (Gudi et al. 2011), has been localized to the acroplaxome, marginal ring, manchette and centrosome during spermatogenesis (Liska et al. 2009). A recent study demonstrated a specific localization for centrobin in the cytoplasm outside of the centrosome, which suggest a possible role as a microtubule nucleation site outside of the centrosome (Shin et al. 2015). Centrobin C-terminal truncation results in decapitated sperm with head and tail malformations. The perinuclear ring was ectopic, and the microtubule organization of the manchette was asymmetric. Moreover, the caudal end of the nucleus was constricted at the 
level of the acroplaxome marginal ring, but the head was not elongated as has been seen in many other microtubule-related gene malfunctions. Thus, the centrobin truncation does not block the manchette microtubule formation, but instead centrobin appears to be required for recruitment of KRT5 (keratin 5) to the marginal ring, which may contribute to the sperm head phenotype (Liska et al. 2009). Malfunction of KRT9 (keratin 9 (K9)), a structural component of the perinuclear ring (Mochida et al. 2000a,b), results with ectopic manchette although the head shaping appears unaffected (Rivkin et al. 2005). The manchette is attached to the nucleus, but abnormally placed and therefore appears to be functional for correct head shaping during spermatid elongation. The malformation of the perinuclear ring and manchette microtubules in the KRT9 mouse model may affect the IMT and thus cause the identified phenotype with coiled tails and also influence the observed residual cytoplasm retention, although this may be a secondary effect. Thus, the correct perinuclear ring formation is not crucial for microtubule nucleation, but it is required for correct manchette formation.

\section{The manchette is organized by the perinuclear ring, but nucleated at the centrosomal adjunct?}

Although the exact mechanism of the manchette formation is not evident, the formed microtubules are connected and stabilized by the perinuclear ring (Fig. 1). CLIP-50, a short isoform of CLIP-170, has been localized to the perinuclear ring during manchette formation (Tarsounas et al. 2001). Tarsounas et al. speculated that the manchette microtubules are formed from the perinuclear ring. However, the staining pattern of $\alpha$-tubulin indicates strong staining at the centrosomal side of the developing manchette (Tarsounas et al. 2001). Our experiments show that microtubules can be initially detected close to the basal body (Fig. 1). The microtubule plus-end tracking protein EB3 is localized to the microtubule ends reaching toward the acrosomal region in steps 7-8 murine spermatids (Fig. 1). After formation of the manchette, EB3 concentrates to the perinuclear ring and the microtubules appear to detach from the centrosomal adjunct (Fig. 1). This observation suggests that EB3 may have a role in stabilizing the microtubule plus-ends attached to the perinuclear ring after formation of the manchette. This hypothesis is supported by the localization of microtubule plus-end tracking proteins close to the perinuclear ring end of the manchette in previous studies (Akhmanova et al. 2005, Kierszenbaum et al. 2011a). Thus, we would like to introduce the hypothesis that the manchette microtubules are nucleated from the centrosomal adjunct with plus-end reaching toward the developing perinuclear ring, and the correct formation of the perinuclear ring is crucial for the organization of the manchette.

\section{Manchette removal}

In the mouse, the manchette moves toward the tail neck region around steps 13-14. The zipper-like movement of the manchette has been suggested to contribute to the slight elongation and flattening of the spermatid head (Russell et al. 1991). During the spermatid elongation, the nuclear ring and entire manchette move toward the spermatid tail, coincident with the acrosome expansion. During the zipper-like movement toward the caudal site of the spermatid head, the perinuclear ring constricts resulting in the correct sperm head shape, which is maintained after the chromatin condensation. The possible arrangement of microtubule plus-ends at the perinuclear ring would enable the function of kinesin-2 as a possible molecular motor for the zipper-like movement of the manchette. This movement is compromised, but not completely blocked by the depletion of KIF3A. Thus, the kinesin- 2 motor has a role in the zipperlike motion of the manchette, but other motors also appear to be involved. It is possible that a malfunction in IMT results in abnormal elongation and delay in manchette clearance, because similar elongated manchette phenotypes have been identified in depletion of proteins important for protein transport such as IFT88 (Kierszenbaum et al. 2011a), and CEP131 (Hall et al. 2013, Fig. 2). Depletion of HOOK1, a protein-linking microtubule to endosomal trafficking, also results in elongated cylindrical shape nucleus in spermatozoa (Mendoza-Lujambio et al. 2002). The interaction between HOOK1 and RIMBP3 has been established, which may represent a link between the manchette microtubules and other cellular organelles, e.g., the nuclear envelope (Zhou et al. 2009). Hook1 and Rimbp3 $\mathrm{KO}$ mouse models also present an ectopic manchette and detached sperm tails. However, the staining pattern in the manchette appears somewhat different for HOOK1 and RIMBP3. HOOK1 is present along the manchette and especially in the perinuclear ring, but RIMBP3 is localized in the caudal part of the manchette, which is not connected to the nucleus. Furthermore, the manchette appears to be connected to the nucleus in Hook1 KO mice, which is demonstrated by the elongated and cylindrical head shape. The ectopic organization of the manchette suggests that HOOK1 may rather have a linking role between the manchette microtubules. Overall, depletion of proteins involved in IFT and microtubule organization-related proteins appear to primarily affect the disassembly of the manchette causing abnormal elongation and cylindrical shape of the sperm head.

The microtubule severing appears to take place at the caudal site of the manchette as supported by the evidence of Katanin localization to this part of the manchette and the Katanin 80 mouse model ( $\mathrm{O}^{\prime}$ Donnell et al. 2012). Katanin 80 is a regulatory subunit of the microtubule severing complex, which is important for 

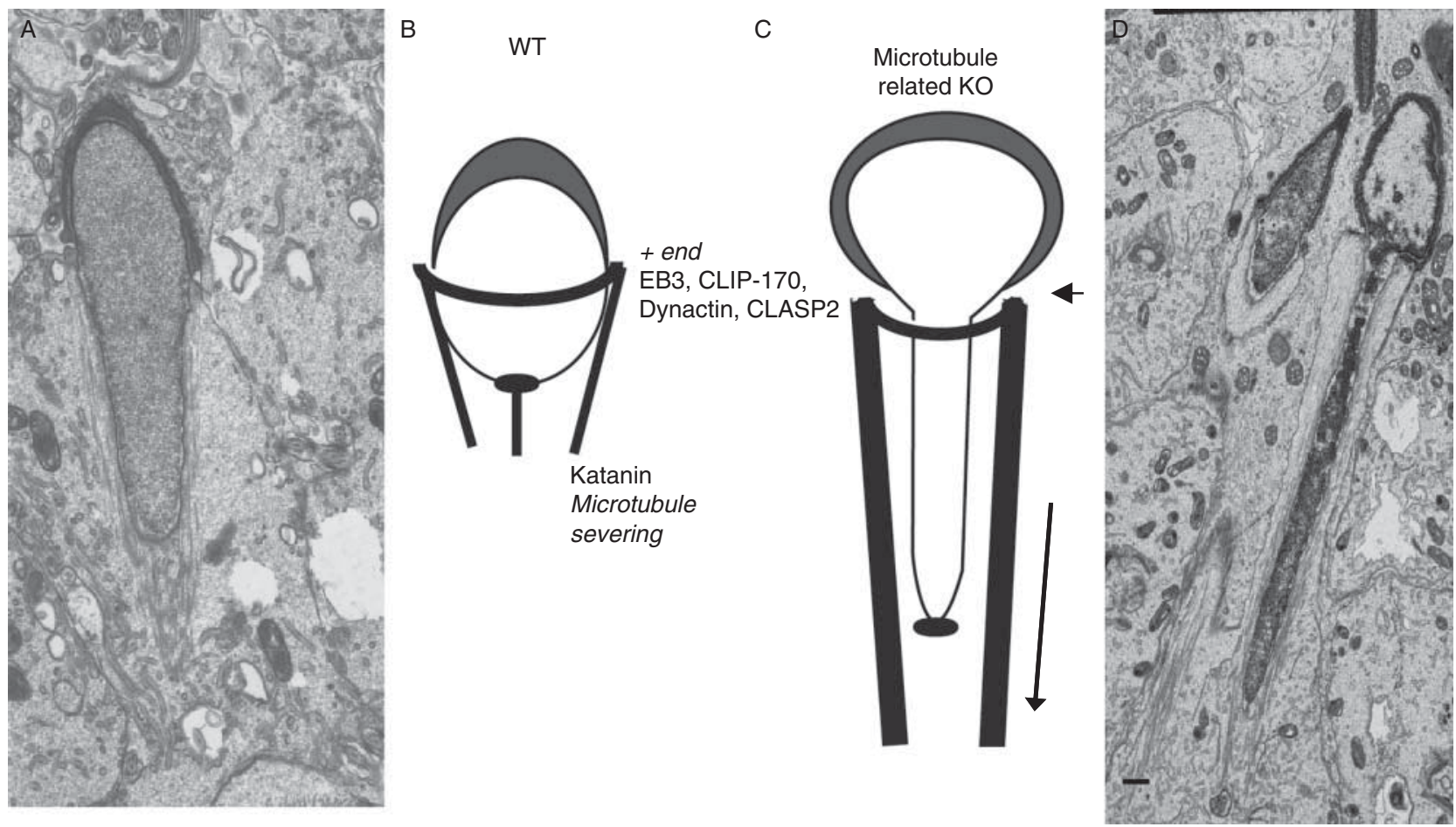

Figure 2 The manchette phenotype of depletion of microtubule-associated proteins. (A) Electron microscopic image of the WT manchette. (B) Schematic presentation of the WT elongating spermatid with the skirt-like manchette and sperm tail. Microtubule plus-end proteins such as EB3, CLIP-170, dynactin and CLASP2 are localized at the perinuclear ring side of the manchette, while microtubule severing enzyme Katanin is localized to the free end of the manchette. (C) Schematic presentation of the elongated spermatid head in microtubule-related mutants. Depletion of, e.g., KIF3A, IFT88, Katanin 80, CEP131, CLIP170 and HOOK1 results in abnormal elongation (arrow) of the manchette. The perinuclear ring (arrowhead) constricts during the zipper-like movement of the manchette during normal sperm head formation. In mutants with elongated manchette, the zipperlike movement is delayed, which result in cylindrical shape of the sperm head. (D) Electron microscopic image of the Kif3A KO spermatid head with elongated manchette.

microtubule dynamics. Katanin 80 stabilizes Katanin 60, which is the catalytic subunit of the complex. Both proteins are localized to the manchette, in particular the caudal end of the microtubules. As microtubule severing is required for deflagellation and microtubule degradation, it can be assumed that the manchette elongation in depletion of Katanin is caused by malfunction of the microtubule severing at the caudal end of the manchette. The lack of Katanin 80 function results in delayed manchette removal and malformation in the sperm tail structures (O'Donnell et al. 2012).

Even though a number of studies have provided observations on the assembly of the manchette, the nucleation sites and exact mechanisms involved remain unclear. The delay in manchette clearance appears in depletion of the anterograde IFT motor protein KIF3A, which suggests that disrupted plus-end directed movement hinders the manchette clearance. On the other hand, the depletion of dynein-related proteins (CLIP-170) result in defects of nuclear shaping. Thus, the established presence of the dynein motor in the connection of the manchette and nucleus (Hayasaka et al. 2008) may contribute to the zipper-like movement together with other molecular motors such as KIFC1 (Yang et al. 2006, Wang et al. 2010). The interaction between KIFC1 and nucleoporin protein NUP62 indicate a role for KIFC1 in nucleocytoplasmic transport, but the decrease in this transport system during nuclear condensation may suggest a more structural role for this complex at the time of manchette removal. Nevertheless, results from mouse models related to the delays in manchette clearance have underlined the importance of correct disassembly and zipper-like movement to sperm head formation. Mouse models affecting the manchette formation are presented in Table 1.

\section{The manchette is connected to the nucleus through the linker of nucleoskeleton and cytoskeleton complex}

Electron microscopy studies have shown that rod-like elements link the manchette microtubules to the nuclear membrane (Russell et al. 1991). The manchette appears to be connected to the nucleoskeleton through the linker of nucleoskeleton and cytoskeleton (LINC) complex (Gob et al. 2010). In somatic cells, the LINC complex 
Table $1 \mathrm{KO}$ mouse models affecting the manchette formation in elongating spermatids.

\begin{tabular}{|c|c|c|c|c|c|}
\hline Gene & Protein & Spermiogenesis phenotype & Interactions & $\begin{array}{l}\text { Identified or suggested function in } \\
\text { somatic cells }\end{array}$ & Reference \\
\hline Azi1/Cep131 & CEP131 & $\begin{array}{l}\text { Short tail, disorganized sperm tail } \\
\text { structures, ectopic and elongated } \\
\text { manchette }\end{array}$ & BBS4 & $\begin{array}{l}\text { Conserved, but non-essential traffick- } \\
\text { ing role in ciliogenesis, localizes } \\
\text { to centriolar satellites and the } \\
\text { transition zone and traffics along } \\
\text { microtubules }\end{array}$ & Hall et al. (2013) \\
\hline Cntrob & CNTROB & $\begin{array}{l}\text { Ectopic and asymmetric perinuclear } \\
\text { ring and manchette, detached } \\
\text { centrosome, decapitated and } \\
\text { disorganized tails }\end{array}$ & KRT5, tubulin & $\begin{array}{l}\text { Required for centriole duplication } \\
\text { and cytokinesis }\end{array}$ & Liska et al. (2009)) \\
\hline E-Map-115 & E-MAP-115 & $\begin{array}{l}\text { Ectopic manchette along regions of } \\
\text { the nucleus that normally do not } \\
\text { display manchette and tail appears } \\
\text { normal }\end{array}$ & Kinesin 1 & Stabilizing and reorganizing MTs & $\begin{array}{l}\text { Komada et al. } \\
\text { (2000)) }\end{array}$ \\
\hline Fused/Axin1 & FU & $\begin{array}{l}\text { Periaxonemal abnormalities, } \\
\text { manchette elongated and } \\
\text { malformed, acroplaxome affected }\end{array}$ & KIF27, ODF1 & $\begin{array}{l}\text { Constructing or maintaining the } \\
\text { central pair apparatus of the } \\
\text { vertebrate } 9+2 \text { axoneme in } \\
\text { multi-ciliated tissues }\end{array}$ & $\begin{array}{l}\text { Nozawa et al. } \\
\quad(2014)\end{array}$ \\
\hline Gорс & GOPC & $\begin{array}{l}\text { Lack of the acrosome, lack of post- } \\
\text { acrosomal sheath and the posterior } \\
\text { ring, misplaced perinuclear ring, } \\
\text { ectopic and misplaced manchette. } \\
\text { Impaired mitochondrial sheath } \\
\text { assembly in the epididymal } \\
\text { spermatozoa, coiled flagella }\end{array}$ & $\begin{array}{l}\text { Golgi-160, } \\
\text { RAB6A, } \\
\text { GRID2, } \\
\text { BECN1, } \\
\text { RHOQ, } \\
\text { ACCN3, } \\
\text { CFTR, } \\
\text { CSPG5 }\end{array}$ & $\begin{array}{l}\text { Trafficking of a subset of plasma } \\
\text { membrane proteins }\end{array}$ & $\begin{array}{l}\text { Suzuki-Toyota } \\
\text { et al. (2004, } \\
\text { 2007) }\end{array}$ \\
\hline Hook1 & HOOK1 & $\begin{array}{l}\text { Manchette elongated, knobbed-like } \\
\text { shape of the head, possibly weak } \\
\text { head tail connection and bending } \\
\text { of the tail }\end{array}$ & RIMBP3 & $\begin{array}{l}\text { Endocytic membrane trafficking } \\
\text { to the microtubule cytoskeleton }\end{array}$ & $\begin{array}{l}\text { Mochida et al. } \\
\text { (1999) }\end{array}$ \\
\hline Ift88 & IFT88 & $\begin{array}{l}\text { No axoneme, disorganized tail } \\
\text { components, malformed HTCA, } \\
\text { ectopic perinuclear ring and } \\
\text { manchette elongated }\end{array}$ & GMAP210 & Part of IFT complex B & $\begin{array}{l}\text { Kierszenbaum } \\
\text { et al. }(2011 b)\end{array}$ \\
\hline $\operatorname{lqcg}$ & IQCG & $\begin{array}{l}\text { Short tail and disorganized sperm tail } \\
\text { structures, irregular nucleus and } \\
\text { localized to the manchette }\end{array}$ & Calmodulin & $\begin{array}{l}\text { Not known and expressed in motile } \\
\text { cilia }\end{array}$ & Harris et al. (2014) \\
\hline Katnb1 & KATNB1 & $\begin{array}{l}\text { Sperm tail motility affected, man- } \\
\text { chette elongated and knobbed-like }\end{array}$ & Katanin 60 & MT severing & $\begin{array}{l}\text { O'Donnell et al. } \\
\text { (2012) }\end{array}$ \\
\hline Kif3A & KIF3A & $\begin{array}{l}\text { No axoneme, disorganized tail } \\
\text { components, manchette elongated } \\
\text { and knobbed-like shape of the head }\end{array}$ & $\begin{array}{l}\text { KIF3B, KAP, } \\
\text { MNS1, KBP }\end{array}$ & IFT anterograde motor & Lehti et al. (2013) \\
\hline Lrguk1 & LRGUK1 & $\begin{array}{l}\text { Short tail, acrosome and acroplaxome } \\
\text { detached, manchette MTs unevenly } \\
\text { distributed and elongated } \\
\text { manchette }\end{array}$ & HOOK2 & Role in MT organization & Liu et al. (2015) \\
\hline Meig1 & MEIG1 & $\begin{array}{l}\text { Disorganized sperm tail structures, } \\
\text { disrupted manchette structure } \\
\text { reported and round or detached } \\
\text { heads }\end{array}$ & $\begin{array}{l}\text { PACRG, } \\
\text { SPAG16 }\end{array}$ & Regulation of meiosis & $\begin{array}{l}\text { Zhang et al. } \\
\qquad(2004,2006)\end{array}$ \\
\hline Pacrg & PACRG & $\begin{array}{l}\text { Disorganized sperm tail structures, } \\
\text { disrupted manchette structure } \\
\text { reported and round or detached } \\
\text { heads }\end{array}$ & MEIG1 & $\begin{array}{l}\text { Localize to cilia and required for } \\
\text { motile cilia function }\end{array}$ & Li et al. (2015) \\
\hline Spef2 & SPEF2 & $\begin{array}{l}\text { Short tail, elongated manchette and } \\
\text { disorganized sperm tail structures }\end{array}$ & IFT20 & $\begin{array}{l}\text { Spef } 2 \mathrm{KO} \text { affects the motile cilia } \\
\text { motion }\end{array}$ & $\begin{array}{l}\text { Sironen et al. } \\
\quad(2010,2011)\end{array}$ \\
\hline Spem 1 & SPEM1 & $\begin{array}{l}\text { Head bend back, mid-piece wrapped } \\
\text { around head and retained } \\
\text { cytoplasm }\end{array}$ & $\begin{array}{l}\text { RANBP17, } \\
\text { UBQLN1 }\end{array}$ & Nucleocytoplasmic transport & $\begin{array}{l}\text { Zheng et al. (2007) } \\
\text { andBao et al. } \\
\text { (2010) }\end{array}$ \\
\hline Sun4 & SUN4 & $\begin{array}{l}\text { Round-headed sperm, severely } \\
\text { disorganized manchette and } \\
\text { coiled tails }\end{array}$ & $\begin{array}{l}\text { SUN3, } \\
\text { Nesprin } 1\end{array}$ & $\begin{array}{l}\text { Expressed only in spermatids and } \\
\text { part of the LINC complex }\end{array}$ & Pasch et al. (2015) \\
\hline Ube $2 b$ & UBE2B & $\begin{array}{l}\text { Mislocation of the longitudinal } \\
\text { columns of the FS, head shape and } \\
\text { MS abnormalities, acrosomal } \\
\text { defects and ectopic manchette }\end{array}$ & RAD18 & $\begin{array}{l}\text { Ubiquitin pathway and protein } \\
\text { degradation }\end{array}$ & $\begin{array}{l}\text { Roest et al. (1996) } \\
\text { and Escalier } \\
\text { et al. (2003) }\end{array}$ \\
\hline
\end{tabular}

$\mathrm{MT}=$ microtubule. 
consists of conserved KASH-SUN protein bridges. Five of the known KASH proteins (Nesprins 1, 2, 3, 4 and KASH5) are outer nuclear membrane adapters for cytoskeletal components and are reported to interact, e.g., with microtubule motors, actin and intermediate filaments (Luxton \& Starr 2014). KASH proteins interact with SUN proteins located in inner nuclear membrane, and this bridge is essential for coupling nuclear structure to cytoskeleton (Crisp et al. 2006). Two LINC complexes have been implicated during spermatid elongation; SUN3/Nesprin 1 complex connects the manchette to the nucleus, and SUN1/Nesprin 3 shows an atypical non-nuclear localization at the anterior pole (Gob et al. 2010). SUN3 and Nesprin 1 have been localized at the sites where the manchette microtubules contact the nuclear envelope (Gob et al. 2010, Calvi et al. 2015). Recently SUN4 has been shown to colocalize with SUN3 and Nesprin 1 (Pasch et al. 2015). SUN4 appears to have the capacity to interact with SUN3 and Nesprin 1 and thus has been suggested to form a complex, which is required for SUN3/Nesprin 1 localization between the manchette and nucleus. In addition, the SUN1/Nesprin 3 localization appears to be wide spread in depletion of SUN4, which underlines the importance of correct formation of the SUN3/Nesprin 1 complex for precise localization of SUN1/Nesprin 3 at the posterior nuclear envelope (Pasch et al. 2015). Interestingly, the SUN3/ Nesprin 1 appears to function in manchette/nucleus connection, and SUN1/Nesprin 3 may have a role in basal body attachment to the nucleus. Interaction between Nesprin 1 and the manchette may occur through the microtubule motors dynein or kinesin or via F-actin. The localization of the cytoplasmic dynein close to the nuclear envelope (Hayasaka et al. 2008) underlines dynein as a candidate motor protein for the LINC complex. LINC complex has been demonstrated to be involved in nuclear shaping, suggesting a possible involvement in spermatid head modeling.

\section{Dynein as the motor between the manchette and nucleus}

The presence of dynein in the manchette has been postulated (Yoshida et al. 1994, Hayasaka et al. 2008). Cytoplasmic dynein was localized to the manchette, close to nuclear envelope at early steps of manchette formation in rats (steps 7-13), with intense staining observed during the most active steps 11-13 of nuclear shaping. Dynamic studies of CLIP-170, a member of the dynein-dynactin pathway, suggest that the dynein complex is a stable component of the manchette (Akhmanova et al. 2005). Cross-sectioned manchette microtubules in Clip-170 KO mice show a highly irregular arrangement, which may indicate that the formation of cross-bridges between microtubules is affected (Akhmanova et al. 2005). These results indicate a possible role in nuclear shaping rather than in IMT.

Some indications of dynein involvement in protein transport have arisen by results from interacting proteins. A protein of the dynactin complex, ARP1, has been localized to the manchette in addition to the centrosome and Golgi complex (Fouquet et al. 2000). ARP1 binds both dynein and cargos (Cheong et al. 2014). ADPribosylation factor-like 3 (ARL3) and dynein light chain LC8 induce dissociation of dynactin from dynein (Jin et al. 2014). A decrease of Arl3 introduced by siRNA injection induced some abnormalities in head shape, lasso-like coiled tail or decapitation (Qi et al. 2013). The lissencephaly protein LIS1 regulates the dynein motility on microtubules (Wang et al. 2013, Toba et al. 2015). It has been suggested that LIS1 may have a role in nuclear condensation based on the mutant phenotype (Nayernia et al. 2003). LIS1 colocalized with dynein along the manchette in elongating spermatids, but not in the axoneme. Furthermore, the acrosome and sperm tail formation was affected. A direct cause and effect of dynein depletion on manchette formation, IMT or nuclear shaping has yet to be established. Although the exact role of dynein motor within the manchette has not been established, it appears to function in connecting the cytoskeleton (manchette) and nucleoskeleton through the LINC complex and may participate in nucleocytoplasmic transport.

\section{The manchette/nucleus connection is crucial for sperm head shaping}

The importance of the manchette in sperm head shaping has become evident during recent years. In general, the spermatid nucleus assumes a parallel shape to the manchette, and when the manchette is absent, the nucleus bulges as a round form as seen in models with caudally displaced manchette (Russell et al. 1991) and in mutants with lack of the nucleus manchette connection (Calvi et al. 2015). When the manchette is abnormally elongated as in many mouse models bearing mutations in IFT or microtubule-related proteins (e.g. KIF3A, IFT88, CLIP-170, HOOK1, FU, KATNB1, PARCG and CEP131), the nucleus is deformed along the elongated manchette as a cylindrical shape (Fig. 2). The connection between the nucleus and the manchette appears unaffected in these mutants causing the elongation of the head along with the manchette. However, the zipper-like movement of the manchette appears affected in these mutants.

The importance of the manchette-nucleus connection has been further demonstrated by the testis-specific LINC component Sun4 KO (Calvi et al. 2015, Pasch et al. 2015). In depletion of SUN4, the manchette microtubules are not laterally associated with nuclear envelope causing round headed sperm. Defects appeared during sperm head condensation, when the 
acrosome was extended to abnormally large area of the nucleus surface and the manchette was highly disorganized. However, chromatin condensation appeared normal in Sun4 KO mice suggesting that abnormal manchette was the major cause for the defects in head shaping. Round-headed sperm has also been detected in Golgi-associated PDZ- and coiled-coil motif-containing protein (GOPC)-deficient mice. Manchette microtubules appeared to be attached to the perinuclear ring, but the manchette and perinuclear ring were placed ectopically, and microtubules were assembled in various directions. In addition, occasional invaginations of the manchette in to the nucleus were observed (Ito et al. 2004). The connection between the nucleus and manchette is crucial for the shaping of the spermatid head, which is demonstrated by the round-headed shape in lack of this connection.

\section{Nucleocytoplasmic transport and protein degradation}

The manchette is involved in transport in and out of the nucleus (nucleocytoplasmic transport) (Kierszenbaum 2002, Kierszenbaum \& Tres 2004). Association of KIFC1 motor with the nucleoporin NUP62 suggests a role for this motor in nucleocytoplasmic transport. Prior to localization to the manchette, KIFC1 is associated with vesicles between the Golgi and the spermatid nucleus and in the acrosome. The integrity of the NUP62 complex is dependent on GTP hydrolysis and the GTP state of the small GTPase Ran (Yang et al. 2006). RAN is required for transport of proteins in and out of the nucleus and for assembly of microtubules (Kierszenbaum et al. 2002, Hermo et al. 2010). RAN and a member of RAN-binding protein RANBP17 are localized along the manchette microtubules. RANBP17 is a potential RAN-dependent importin for protein transport in and out of the nucleus. RANBP17 appears to interact with SPEM1, the depletion of which causes bent heads and remnant cytoplasm in mature sperm (Zheng et al. 2007). The 'head-bent-back' sperm phenotype has been observed in many mouse lines with targeted inactivation of proteins involved in nuclear condensation (Xu et al. 1999, Adham et al. 2001, Cho et al. 2001, Tanaka et al. 2005). However, the sperm head condensation and tail structures appeared unaffected in Spem1 KO mice, which suggests that SPEM1/ RANBP17 interaction may have a role in nucleocytoplasmic transport required for correct removal of the cytoplasm. An additional interacting partner, UBQLN1, has also been proposed for SPEM1. UBQLN1 is part of the ubiquitin-proteosome (UBP) system, which degrades unwanted proteins. Although the exact role for SPEM1 is not known, it may have a role in UBP system through the interactions with UBQLN1. Mutation in murine ubiquitin conjugating enzyme mHR6B (UBE2B) causes heterogeneous malformations in head shaping and sperm tail formation. In Ube $2 b$ KO mouse, the manchette was ectopic with microtubule invaginations within the nucleus. Membranous structures were associated with the microtubules, which could represent a possible defect in the transport of vesicular material along the manchette (Escalier et al. 2003). This mouse model also underlines the functional involvement of the UBP in the assembly of flagellar components in mammals. The role of the UBP system within the manchette is further supported by the localization of an ubiquitin protein ligase RNF19A and components of $26 \mathrm{~S}$ proteosome (e.g. PSMC3 and TBP-1) (Mochida et al. 2000a, b, Rivkin et al. 2009). The evidence points toward a role for the manchette in Ran GTPase-mediated nucleocytoplasmic trafficking of proteins for disposal by the manchette-associated $26 \mathrm{~S}$ proteasome. The manchette appears to have a role in regulating protein quality and nucleocytoplasmic transport. However, little is known about the activity of these pathways during spermatid elongation.

\section{IMT delivers proteins during spermatid elongation}

IMT is carried out by molecular motors, kinesins and dynein, along the microtubule tracks and by myosin along the actin tracks within the manchette. F-actinrelated transport proteins myosin VA, MyRIP and RAB27B have been localized to the sperm manchette in humans (Kierszenbaum et al. 2003, Hayasaka et al. 2008) and may contribute to the short distance transport of proteins. The long distance transport has been suggested to be implemented by the microtubule tracks (Goode et al. 2000). It has been hypothesized that proteins can switch between these two types of transport mechanisms (Kierszenbaum \& Tres 2004), and the co-operation results in timely delivery of proteins (Gross et al. 2000). The suggestion that IMT has molecular homology with IFT (Kierszenbaum 2002) is supported by investigations based on protein localizations and $\mathrm{KO}$ mouse models of known IFT proteins KIF3A, IFT20, IFT88 and KIF27 (Sironen et al. 2010, Kierszenbaum et al. 2011b, Lehti et al. 2013, Nozawa et al. 2014). All IFT proteins are expressed in spermatogenesis, and the expression appears to increase during the progress of sperm development (Fig. 3). Although the expression indicates that the protein products are required for sperm development, the exact roles for most of the IFT proteins are not known. In addition, KIF17B (Saade et al. 2007) and KIFC5A (Navolanic \& Sperry 2000) have been associated with the manchette and may contribute to the IMT. KIF17B has been shown to interact with its cargo protein spatial in the manchette and also along the sperm tail principal piece in mature sperm (Saade et al. 2007). 

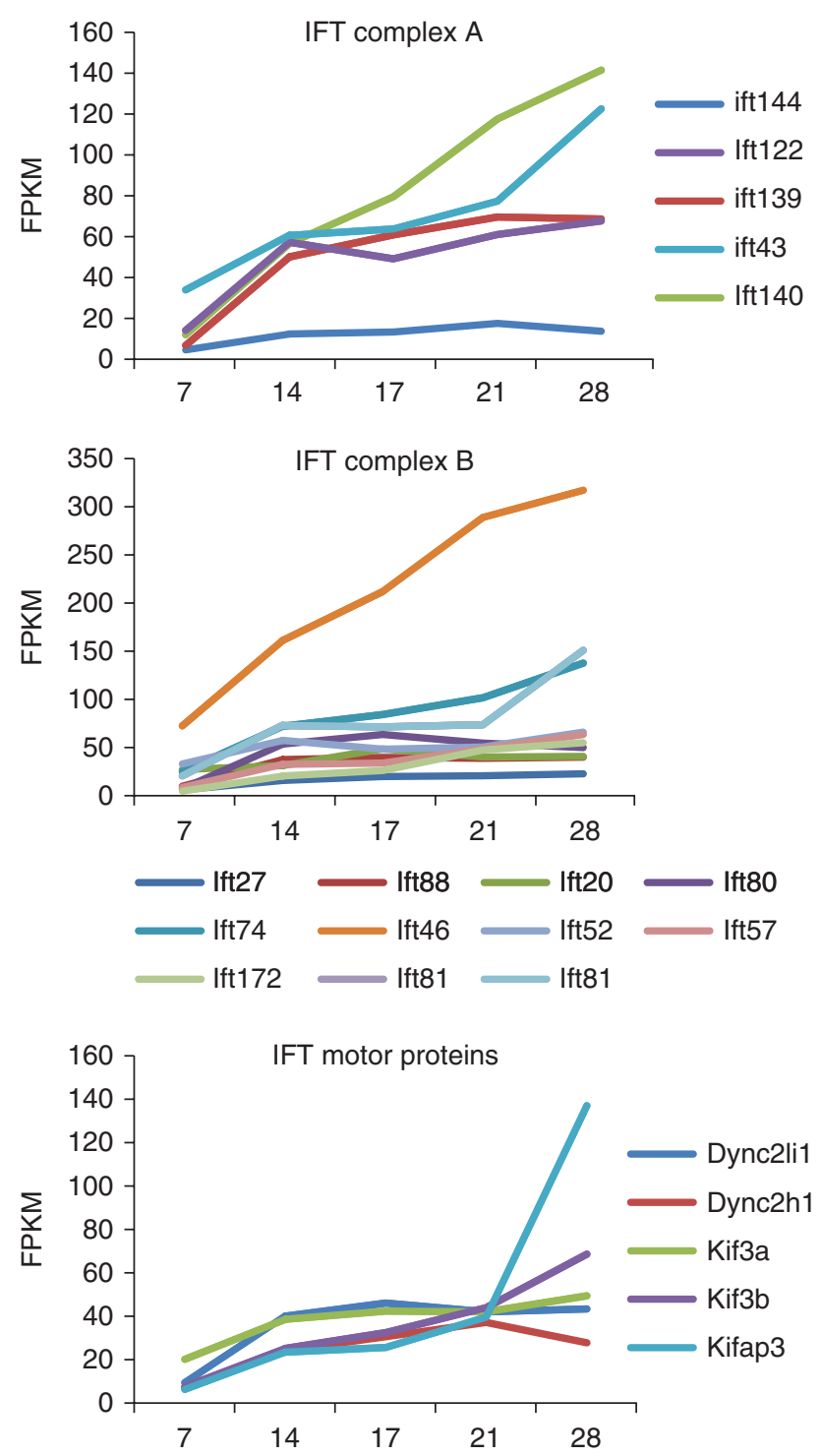

Figure 3 IFT genes are expressed during spermatogenesis. IFT motor proteins and complex $A$ and $B$ genes have increasing expression pattern during the first wave of spermatogenesis based on transcriptomic sequencing data (Laiho et al. 2013). The $X$-axis shows the postnatal development days.

\section{Kinesin-2 and IFT complex B proteins are present in the manchette}

The Kif3A KO demonstrated the consequences of IFT motor protein loss during spermatogenesis. In addition to the axoneme formation, KIF3A depletion affects the elongation and clearance of the manchette (Lehti et al. 2013), underlying the importance of kinesin-2 motor to correct head shaping and manchette clearance. A role of kinesin-2 in protein transport through the manchette was also implicated by delayed translocation of MNS1 staining from the acroplaxome to the manchette and finally to the sperm tail. MNS1 depletion only affects sperm tail structures (Zhou et al. 2012) suggesting that
MNS1 is a cargo particle of the IMT and transported through the manchette to the developing sperm tail (Lehti et al. 2013). Two other IFT complex B proteins, IFT88 and IFT20, have also been localized to the manchette. Studies with the Ift $88 \mathrm{KO}$ mouse model provided further evidence of the involvement of IFT in spermatid nuclear shaping and development of the centrosome-derived head-tail coupling apparatus and sperm tail (San Agustin et al. 2015). The phenotypes in Kif3A and Ift88 $\mathrm{KO}$ mouse models were similar, supporting the hypothesis of protein delivery via IMT through the recruitment of IFT complex B.

\section{Delivery of sperm tail proteins through the manchette}

IMT has been demonstrated to store and deliver structural sperm tail proteins to the basal body region (Kierszenbaum 2001). Depletion of IFT88 results in the accumulation of a GMAP210-stained vesicle with mitochondrial sheath and outer dense fiber (ODF) material in the manchette, indicative of defects in protein delivery (Kierszenbaum et al. 2011a, Fig. 4). In Ift88 KO mice, myosin VA appeared to accumulate in the caudal site of the manchette indicating that inhibition of microtubule-based transport may affect the F-actin motor proteins (Kierszenbaum et al. 2011b). This observation also further supports the hypothesis that the microtubule plus-end is in fact at the perinuclear ring of the manchette. A protein required for the central pair assembly in motile cilia, the putative serine-threonine kinase fused (FU), localizes to the manchette and the acrosome-acroplaxome. The depletion of FU affects the formation of sperm tail accessory structures, but the axoneme formation appears unaffected; thus, FU appears to have a slightly different role in sperm tail development than in motile cilia. FU interacts with ODF1 and KIF27 (Nozawa et al. 2014) and thus may be transported through the manchette to the developing sperm tail. KIF27-GFP localizes to the perinuclear ring of the manchette and with a weaker signal to the manchette in elongating spermatids. Furthermore, the sperm flagellar 2 (SPEF2) protein, which is required for cilia motility and formation of sperm tail structures, is present in the manchette prior to the localization to the sperm tail mid-piece (Sironen et al. 2010, 2011). The interaction between SPEF2 and IFT20 has been postulated; however, it is not clear if SPEF2 is transported through the manchette or if it has a role in the IMT. Nevertheless, the recent findings suggest a role of IFT complex B in transport of sperm tail components through the manchette to the base of the sperm tail (Fig. 4).

The depletion of HOOK1 protein results in elongated manchette and malformed attachment of the connecting piece (implantation fossa) and sperm tail mid-piece anomalies (Mochida et al. 1999, Mendoza-Lujambio et al. 2002). In addition to the possible microtubulelinking properties of HOOK1, it may function in protein 


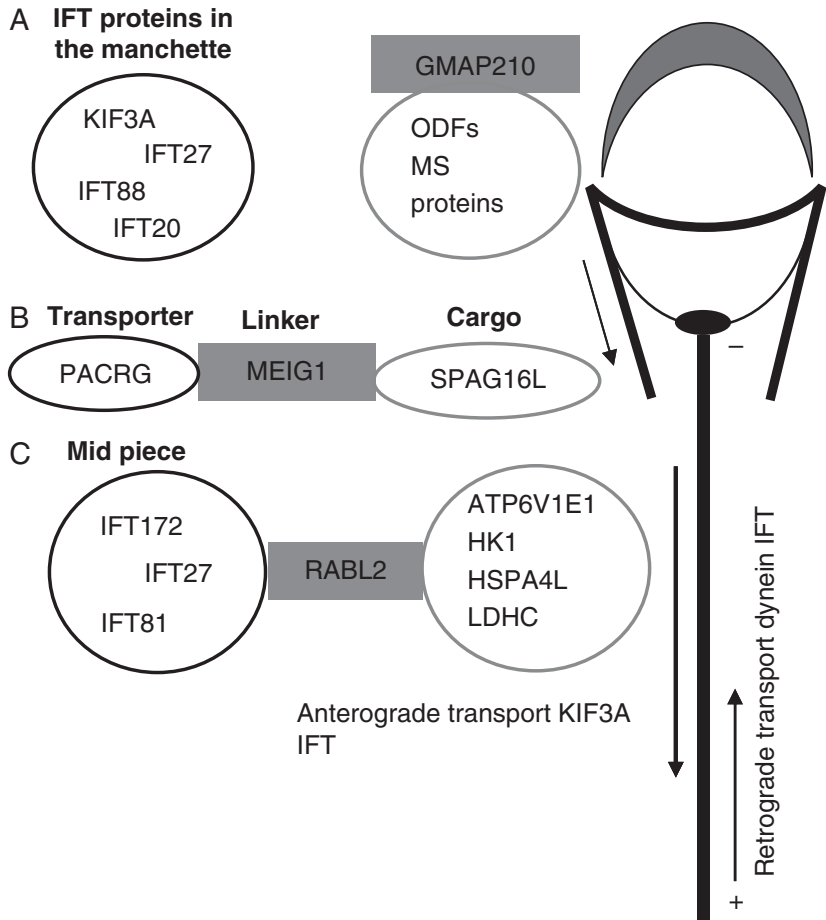

Figure 4 Identified IFT-related transport complexes during spermatid elongation. (A) KIF3A, IFT88, IFT27 and IFT20 are localized to the spermatid manchette and based on the KO mouse model affect delivery of proteins to the sperm tail. GMAP210 interacts with cargo protein vesicles, which transport to the developing sperm tail is blocked in depletion of IFT88. (B) PACRG/MEIG1 interaction affects the localization of SPAG16L to the sperm tail. (C) IFT complex B proteins IFT27, 81 and 172 have been localized to the sperm tail mid-piece and through interaction with RABL2 appear to be involved in transporting proteins required for glycolysis to the sperm tail FS. However, not all IFT proteins appear to be present in the mid-piece of the sperm tail; thus, the exact role of IFT during late steps of sperm tail development is not known.

transport to the basal body. HOOK1 has been reported to link the endocytic membrane trafficking to the microtubule cytoskeleton (Maldonado-Baez et al. 2013). The cargo-specific binding properties of HOOK1 and its ability to bind motor proteins may contribute to transport of cargos to specific locations within the elongating spermatid. HOOK1 belongs to HOOK protein family, which is known to function as cargo-loading proteins onto microtubules for transport. The depletion of an interaction partner of another HOOK protein family member, HOOK2, also causes cylindrical head shaping (Liu et al. 2015). The uncharacterized protein called leucine-rich repeats and guanylate kinase domain containing isoform 1 (LRGUK1) is transported via acrosome-acroplaxome-manchette-tail axis in a complex with HOOK2. LRGUK1 dysfunction caused disorganized and uneven distribution of manchette microtubules and lack of the sperm tail axoneme (Liu et al. 2015). The involvement of HOOK2/LRGUK1 complex has been suggested in protein transport, but the exact mechanism is not known. The HOOK family members may represent one cargo-specific link between the motors and cargo within the manchette in addition to acrosome and acroplaxome vesicle transport. However, the elucidation of the exact transport mechanisms and cargo/motor complexes during IMT of sperm tail proteins require extensive further studies.

For IFT proteins, their importance in transport along the developing sperm tail hinders the evaluation of the role of IMT for sperm tail development. However, studies of transport complexes localized only in the manchette underline the crucial importance of IMT for sperm tail development. Meiosis-expressed gene 1 (MEIG1) and Parkin-co-regulated gene (PACRG) form a complex in the manchette, which appears to be required for transport of cargos to the sperm tail (Li et al. 2015). PACRG depletion affects the localization of MEIG1 in the manchette indicating that it is an upstream protein in the pathway. Both Meig1 and Pacrg KO are known to affect the localization of axonemal central apparatus protein SPAG16L suggesting that SPAG16L is simply transported through the manchette to the developing sperm tail. In the Spag16L KO, sperm tail motility is compromised (Zhang et al. 2006).

\section{Protein transport mechanisms during formation of the accessory structures of the sperm tail}

The different components of the sperm tail require ordered delivery of particles to the assembly site. After axoneme formation, all accessory structures need to be compiled. The ODFs are constructed along the axoneme, and fibrous sheath (FS) is built in distal to proximal orientation; thus, the proteins need to be transported all the way to the flagellar tip. However, little is known about the exact delivery systems during sperm tail development. IFT has been suggested to be the delivery mechanism in a similar manner as described for cilia formation. Depletion of the kinesin-2 motor resulted in the inability to construct the sperm tail axoneme (Lehti et al. 2013). It remains unclear if this is the sole transport mechanism required for sperm tail formation. The fact that the FS and ODFs are assembled in opposite directions implies that there are at least two different transport mechanisms. Although various proteins intended for the sperm tail appear to be stored in the manchette prior to transportation to the sperm tail, the FS proteins have not been identified in the manchette. Rabl2 structural mutant has been shown to affect the delivery of several components to the developing sperm tail. It has been suggested that RABL2 interacts with IFT proteins IFT27, IFT172 and IFT81 (Lo et al. 2012), but the possible interaction site was identified in the mid-piece of the sperm tail (Fig. 4). This led the authors to hypothesize that the lack of RABL2 in the mid-piece affects the localization of ATP6V1E1, glycolytic enzyme 
HK1, HSPA4L and lactate dehydrogenase C (LDHC) in the principal piece. Given that the mid-piece is the last structure to appear in the sperm tail, such a transport mechanism is unlikely to be involved in flagella formation, but rather in the delivery of proteins required for sperm motility to the principal piece. Mouse models of HSPA4L and LDHC depletion cause lowered sperm motility without any structural malformations (Held et al. 2006, Odet et al. 2008). Such observations suggest that RABL2 and the interaction with IFT proteins are specifically required for transport of proteins important for motility pathways, probably for glycolysis, in the principal piece of the sperm tail. It should be noted that this is the first study to identify possible transport pathway for principal piece proteins, which may deliver non-structural proteins from the cytoplasm through the mid-piece after formation of the sperm tail. RABL2 may have a role in delivering proteins to the base of the IFT initiation site, which could be demarcated by the septin ring after formation of the mid-piece. This septin ring moves distally from the base of the sperm tail during the mid-piece formation enabling the mitochondria to be laid down along the developing mid-piece. The kinesin-2 motor protein KIF3A has been localized to the FS in addition to the manchette (Lehti et al. 2013), but IFT20 was localized only to the manchette and basal body (Sironen et al. 2010). Thus, not all IFT complex B proteins are localized to the mid-piece or principal piece of the sperm tail. The elucidation of the exact role of IFT and related proteins during late steps of sperm tail development require further studies.

\section{Fertile sperm production requires specialized transport mechanisms}

Male infertility is caused by various defects during the complex process of spermatogenesis. Recent reports highlight the fact that successful sperm production requires proteins and protein complexes to be transported to the correct assembly site during sperm tail formation. However, the exact role of microtubule and actin-based delivery complexes are not well characterized. Some proteins appear to be transported through the acroplaxome and manchette to the base of the developing sperm tail and may be stored transiently in these structures. However, not all sperm tail proteins have been localized to the manchette, raising the prospect that different mechanisms for delayed translation or protein storage and transport are involved. Functional studies need to be undertaken to provide a more complete understanding of the mechanisms involved in fertile sperm formation, which is important for developing therapies for male infertility. This is particularly important, since the genetic alterations may also affect the ICSI outcome and thus be transferred to the next generation. During spermatid elongation, the manchette is a crucial structure orchestrating protein distribution and function. Sperm tail development is dependent on both IMT and IFT, with a common molecular system contributing to both transport mechanisms. Although recent studies have shown that disruption of the protein delivery to the correct assembly site during spermiogenesis results in detrimental malformations in mature sperm, the exact mechanism by which the specific cargos are stored, recognized and finally delivered is not known. Thus, to unravel the possible causes for male infertility, we need to investigate the exact mechanism involved in these specific events during spermatid elongation.

\section{Declaration of interest}

The authors declare that there is no conflict of interest that could be perceived as prejudicing the impartiality of the review.

\section{Funding}

This work was supported by the Academy of Finland, Häme Regional Fund of Finnish Cultural foundation and Turku Doctoral Programme of Molecular Medicine.

\section{References}

Adham IM, Nayernia K, Burkhardt-Gottges E, Topaloglu O, Dixkens C, Holstein AF \& Engel W 2001 Teratozoospermia in mice lacking the transition protein 2 (Tnp2). Molecular Human Reproduction 7 513-520. (doi:10.1093/molehr/7.6.513)

Akhmanova A, Mausset-Bonnefont AL, van Cappellen W, Keijzer N, Hoogenraad CC, Stepanova T, Drabek K, van der Wees J, Mommaas M, Onderwater J et al. 2005 The microtubule plus-end-tracking protein CLIP-170 associates with the spermatid manchette and is essential for spermatogenesis. Genes \& Development 19 2501-2515. (doi:10.1101/ $\operatorname{gad} .344505)$

Baldari CT \& Rosenbaum J 2010 Intraflagellar transport: it's not just for cilia anymore. Current Opinion in Cell Biology 22 75-80. (doi:10.1016/j.ceb. 2009.10.010)

Bao J, Zhang J, Zheng H, Xu C \& Yan W 2010 UBQLN1 interacts with SPEM1 and participates in spermiogenesis. Molecular and Cellular Endocrinology 327 89-97. (doi:10.1016/j.mce.2010.06.006)

Calvi A, Wong AS, Wright G, Wong ES, Loo TH, Stewart CL \& Burke B 2015 SUN4 is essential for nuclear remodeling during mammalian spermiogenesis. Developmental Biology 407 321-330. (doi:10.1016/j.ydbio. 2015.09.010)

Cheong FK, Feng L, Sarkeshik A, Yates JRIII \& Schroer TA 2014 Dynactin integrity depends upon direct binding of dynamitin to Arp1. Molecular Biology of the Cell 25 2171-2180. (doi:10.1091/mbc.E14-03-0842)

Cho C, Willis WD, Goulding EH, Jung-Ha H, Choi YC, Hecht NB \& Eddy EM 2001 Haploinsufficiency of protamine-1 or -2 causes infertility in mice. Nature Genetics 28 82-86.

Crisp M, Liu Q, Roux K, Rattner JB, Shanahan C, Burke B, Stahl PD \& Hodzic D 2006 Coupling of the nucleus and cytoplasm: role of the LINC complex. Journal of Cell Biology 172 41-53. (doi:10.1083/jcb. 200509124)

Escalier D, Bai XY, Silvius D, Xu PX \& Xu X 2003 Spermatid nuclear and sperm periaxonemal anomalies in the mouse Ube $2 \mathrm{~b}$ null mutant. Molecular Reproduction and Development 65 298-308. (doi:10.1002/ mrd.10290) 
Fouquet J, Kann M, Soues S \& Melki R 2000 ARP1 in Golgi organisation and attachment of manchette microtubules to the nucleus during mammalian spermatogenesis. Journal of Cell Science 113 877-886.

Gob E, Schmitt J, Benavente R \& Alsheimer M 2010 Mammalian sperm head formation involves different polarization of two novel LINC complexes. PloS ONE 5 e12072. (doi:10.1371/journal.pone.0012072)

Goode BL, Drubin DG \& Barnes G 2000 Functional cooperation between the microtubule and actin cytoskeletons. Current Opinion in Cell Biology 12 63-71. (doi:10.1016/S0955-0674(99)00058-7)

Gross SP, Welte MA, Block SM \& Wieschaus EF 2000 Dynein-mediated cargo transport in vivo. A switch controls travel distance. Journal of Cell Biology 148 945-956. (doi:10.1083/jcb.148.5.945)

Gudi R, Zou C, Li J \& Gao Q 2011 Centrobin-tubulin interaction is required for centriole elongation and stability. Journal of Cell Biology 193 711-725. (doi:10.1083/jcb.201006135)

Hall EA, Keighren M, Ford MJ, Davey T, Jarman AP, Smith LB, Jackson IJ \& Mill P 2013 Acute versus chronic loss of mammalian Azi1/Cep131 results in distinct ciliary phenotypes. PLoS Genetics 9 e1003928. (doi:10.1371/journal.pgen.1003928)

Harris TP, Schimenti KJ, Munroe RJ \& Schimenti JC 2014 IQ motifcontaining G (Iqcg) is required for mouse spermiogenesis. G3 4 367-372. (doi:10.1534/g3.113.009563)

Hayasaka S, Terada Y, Suzuki K, Murakawa H, Tachibana I, Sankai T, Murakami T, Yaegashi N \& Okamura K 2008 Intramanchette transport during primate spermiogenesis: expression of dynein, myosin $\mathrm{Va}$, motor recruiter myosin Va, VIla-Rab27a/b interacting protein, and Rab27b in the manchette during human and monkey spermiogenesis. Asian Journal of Andrology 10 561-568. (doi:10.1111/j.1745-7262.2008. 00392.x)

Held T, Paprotta I, Khulan J, Hemmerlein B, Binder L, Wolf S, Schubert S, Meinhardt A, Engel W \& Adham IM 2006 Hspa4l-deficient mice display increased incidence of male infertility and hydronephrosis development. Molecular and Cellular Biology 26 8099-8108. (doi:10.1128/MCB. 01332-06)

Hermo L, Pelletier RM, Cyr DG \& Smith CE 2010 Surfing the wave, cycle, life history, and genes/proteins expressed by testicular germ cells. Part 2: changes in spermatid organelles associated with development of spermatozoa. Microscopy Research and Technique 73 279-319. (doi:10.1002/jemt.20783)

Ishihara K, Nguyen PA, Groen AC, Field CM \& Mitchison TJ 2014 Microtubule nucleation remote from centrosomes may explain how asters span large cells. PNAS 111 17715-17722. (doi:10.1073/pnas. 1418796111)

Ito C, Suzuki-Toyota F, Maekawa M, Toyama Y, Yao R, Noda T \& Toshimori K 2004 Failure to assemble the peri-nuclear structures in GOPC deficient spermatids as found in round-headed spermatozoa. Archives of Histology and Cytology 67 349-360. (doi:10.1679/aohc.67.349)

Jin M, Yamada M, Arai Y, Nagai T \& Hirotsune S 2014 Arl3 and LC8 regulate dissociation of dynactin from dynein. Nature Communications $\mathbf{5}$ 5295. (doi:10.1038/ncomms6295)

Kierszenbaum AL 2001 Spermatid manchette: plugging proteins to zero into the sperm tail. Molecular Reproduction and Development 59 347-349. (doi:10.1002/mrd.1040)

Kierszenbaum AL 2002 Intramanchette transport (IMT): managing the making of the spermatid head, centrosome, and tail. Molecular Reproduction and Development 63 1-4. (doi:10.1002/mrd.10179)

Kierszenbaum AL \& Tres LL 2004 The acrosome-acroplaxome-manchette complex and the shaping of the spermatid head. Archives of Histology and Cytology 67 271-284. (doi:10.1679/aohc.67.271)

Kierszenbaum AL, Gil M, Rivkin E \& Tres LL 2002 Ran, a GTP-binding protein involved in nucleocytoplasmic transport and microtubule nucleation, relocates from the manchette to the centrosome region during rat spermiogenesis. Molecular Reproduction and Development 63 131-140. (doi:10.1002/mrd.10164)

Kierszenbaum AL, Rivkin E \& Tres LL 2003 The actin-based motor myosin $\mathrm{Va}$ is a component of the acroplaxome, an acrosome-nuclear envelope junctional plate, and of manchette-associated vesicles. Cytogenetic and Genome Research 103 337-344. (doi:10.1159/000076822)

Kierszenbaum AL, Rivkin E \& Tres LL 2011 a Cytoskeletal track selection during cargo transport in spermatids is relevant to male fertility. Spermatogenesis 1 221-230. (doi:10.4161/spmg.1.3.18018)
Kierszenbaum AL, Rivkin E, Tres LL, Yoder BK, Haycraft CJ, Bornens M \& Rios RM $2011 b$ GMAP210 and IFT88 are present in the spermatid golgi apparatus and participate in the development of the acrosomeacroplaxome complex, head-tail coupling apparatus and tail. Developmental Dynamics 240 723-736. (doi:10.1002/dvdy.22563)

Komada M, McLean DJ, Griswold MD, Russell LD \& Soriano P 2000 E-MAP115 , encoding a microtubule-associated protein, is a retinoic acidinducible gene required for spermatogenesis. Genes and Development 14 1332-1342.

Laiho A, Kotaja N, Gyenesei A \& Sironen A 2013 Transcriptome profiling of the murine testis during the first wave of spermatogenesis. PloS ONE $\mathbf{8}$ e61558. (doi:10.1371/journal.pone.0061558)

Lehti MS, Kotaja N \& Sironen A 2013 KIF3A is essential for sperm tail formation and manchette function. Molecular and Cellular Endocrinology 377 44-55. (doi:10.1016/j.mce.2013.06.030)

Li W, Tang W, Teves ME, Zhang Z, Zhang L, Li H, Archer KJ, Peterson DL, Williams DC Jr, Strauss JF III et al. 2015 A MEIG1/PACRG complex in the manchette is essential for building the sperm flagella. Development 142 921-930. (doi:10.1242/dev.119834)

Liska F, Gosele C, Rivkin E, Tres L, Cardoso MC, Domaing P, Krejci E, Snajdr P, Lee-Kirsch MA, de Rooij DG, Kren V, Krenova D, Kierszenbaum AL \& Hubner N 2009 Rat hd mutation reveals an essential role of centrobin in spermatid head shaping and assembly of the head-tail coupling apparatus. Biology of Reproduction 81 1196-1205. (doi:10.1095/biolreprod.109.078980)

Liu Y, DeBoer K, de Kretser DM, O'Donnell L, O'Connor AE, Merriner DJ, Okuda H, Whittle B, Jans DA, Efthymiadis A, McLachlan RI, Ormandy CJ, Goodnow CC, Jamsai D \& O'Bryan MK 2015 LRGUK-1 is required for basal body and manchette function during spermatogenesis and male fertility. PLoS Genetics 11 e1005090. (doi:10.1371/ journal.pgen.1005090)

Lo JC, Jamsai D, O'Connor AE, Borg C, Clark BJ, Whisstock JC, Field MC, Adams V, Ishikawa T, Aitken RJ, Whittle B, Goodnow CC, Ormandy CJ \& O'Bryan MK 2012 RAB-like 2 has an essential role in male fertility, sperm intra-flagellar transport, and tail assembly. PLoS Genetics 8 e1002969. (doi:10.1371/journal.pgen.1002969)

Luxton GW \& Starr DA 2014 KASHing up with the nucleus: novel functional roles of KASH proteins at the cytoplasmic surface of the nucleus. Current Opinion in Cell Biology 28 69-75. (doi:10.1016/j.ceb. 2014.03.002)

Maldonado-Baez L, Cole NB, Kramer H \& Donaldson JG 2013 Microtubule-dependent endosomal sorting of clathrin-independent cargo by Hook1. Journal of Cell Biology 201 233-247. (doi:10.1083/ jcb.201208172)

Mendoza-Lujambio I, Burfeind P, Dixkens C, Meinhardt A, Hoyer-Fender S, Engel W \& Neesen J 2002 The Hook1 gene is non-functional in the abnormal spermatozoon head shape (azh) mutant mouse. Human Molecular Genetics 11 1647-1658. (doi:10.1093/hmg/11.14.1647)

Mochida K, Tres LL \& Kierszenbaum AL 1999 Structural and biochemical features of fractionated spermatid manchettes and sperm axonemes of the azh/azh mutant mouse. Molecular Reproduction and Development 52 434-444. (doi:10.1002/(SICI)1098-2795(199904)52:4<434::AIDMRD13 > 3.0.CO;2-D)

Mochida K, Rivkin E, Gil M \& Kierszenbaum AL 2000a Keratin 9 is a component of the perinuclear ring of the manchette of rat spermatids. Developmental Biology 227 510-519. (doi:10.1006/dbio.2000.9911)

Mochida K, Tres LL \& Kierszenbaum AL $2000 b$ Structural features of the $26 \mathrm{~S}$ proteasome complex isolated from rat testis and sperm tail. Molecular Reproduction and Development 57 176-184. (doi:10.1002/10982795(200010)57:2<176::AID-MRD9>3.0.CO;2-O)

Moreno RD \& Schatten G 2000 Microtubule configurations and posttranslational $\alpha$-tubulin modifications during mammalian spermatogenesis. Cell Motility and the Cytoskeleton 46 235-246. (doi:10.1002/ 1097-0169(200008)46:4 <235::AID-CM1 > 3.0.CO;2-G)

Navolanic PM \& Sperry AO 2000 Identification of isoforms of a mitotic motor in mammalian spermatogenesis. Biology of Reproduction 62 1360-1369. (doi:10.1095/biolreprod62.5.1360)

Nayernia K, Vauti F, Meinhardt A, Cadenas C, Schweyer S, Meyer BI, Schwandt I, Chowdhury K, Engel W \& Arnold HH 2003 Inactivation of a testis-specific Lis1 transcript in mice prevents spermatid differentiation and causes male infertility. Journal of Biological Chemistry 278 48377-48385. (doi:10.1074/jbc.M309583200) 
Nozawa YI, Yao E, Gacayan R, Xu SM \& Chuang PT 2014 Mammalian Fused is essential for sperm head shaping and periaxonemal structure formation during spermatogenesis. Developmental Biology 388 170-180. (doi:10.1016/j.ydbio.2014.02.002)

Odet F, Duan C, Willis WD, Goulding EH, Kung A, Eddy EM \& Goldberg E 2008 Expression of the gene for mouse lactate dehydrogenase $C$ (Ldhc) is required for male fertility. Biology of Reproduction 79 26-34. (doi:10.1095/biolreprod.108.068353)

O'Donnell L \& O'Bryan MK 2014 Microtubules and spermatogenesis. Seminars in Cell \& Developmental Biology 30 45-54. (doi:10.1016/j. semcdb.2014.01.003)

O'Donnell L, Rhodes D, Smith SJ, Merriner DJ, Clark BJ, Borg C, Whittle B, O'Connor AE, Smith LB \& McNally FJ 2012 An essential role for katanin p80 and microtubule severing in male gamete production. PLoS Genetics 8 e1002698. (doi:10.1371/journal.pgen.1002698)

Pasch E, Link J, Beck C, Scheuerle S \& Alsheimer M 2015 The LINC complex component Sun4 plays a crucial role in sperm head formation and fertility. Biology Open 4 1792-1802. (doi:10.1242/bio.015768)

Qi Y, Jiang M, Yuan Y, Bi Y, Zheng B, Guo X, Huang X, Zhou Z \& Sha J 2013 ADP-ribosylation factor-like 3, a manchette-associated protein, is essential for mouse spermiogenesis. Molecular Human Reproduction 19 327-335. (doi:10.1093/molehr/gat001)

Rivkin E, Eddy EM, Willis WD, Goulding EH, Suganuma R, Yanagimachi R \& Kierszenbaum AL 2005 Sperm tail abnormalities in mutant mice with neo(r) gene insertion into an intron of the keratin 9 gene. Molecular Reproduction and Development 72 259-271. (doi:10.1002/mrd.20335)

Rivkin E, Kierszenbaum AL, Gil M \& Tres LL 2009 Rnf19a, a ubiquitin protein ligase, and Psmc3, a component of the $26 \mathrm{~S}$ proteasome, tether to the acrosome membranes and the head-tail coupling apparatus during rat spermatid development. Developmental Dynamics 238 1851-1861. (doi:10.1002/dvdy.22004)

Roest HP, van Klaveren J, de Wit J, van Gurp CG, Koken MH, Vermey M, van Roijen JH, Hoogerbrugge JW, Vreeburg JT, Baarends WM, Bootsma D, Grootegoed JA \& Hoeijmakers JH 1996 Inactivation of the HR6B ubiquitin-conjugating DNA repair enzyme in mice causes male sterility associated with chromatin modification. Cell 86 799-810. (doi:10.1016/S0092-8674(00)80154-3)

Russell LD, Russell JA, MacGregor GR \& Meistrich ML 1991 Linkage of manchette microtubules to the nuclear envelope and observations of the role of the manchette in nuclear shaping during spermiogenesis in rodents. American Journal of Anatomy 192 97-120. (doi:10.1002/aja. 1001920202)

Saade M, Irla M, Govin J, Victorero G, Samson M \& Nguyen C 2007 Dynamic distribution of Spatial during mouse spermatogenesis and its interaction with the kinesin KIF17b. Experimental Cell Research 313 614-626. (doi:10.1016/j.yexcr.2006.11.011)

San Agustin JT, Pazour GJ \& Witman GB 2015 Intraflagellar transport is essential for mammalian spermiogenesis but is absent in mature sperm. Molecular Biology of the Cell 26 4358-4372. (doi:10.1091/mbc. E15-08-0578)

Shen J, Chen W, Shao B, Qi Y, Xia Z, Wang F, Wang L, Guo X, Huang X \& Sha J 2014 Lamin A/C proteins in the spermatid acroplaxome are essential in mouse spermiogenesis. Reproduction 148 479-487. (doi:10.1530/REP-14-0012)

Shin W, Yu NK, Kaang BK \& Rhee K 2015 The microtubule nucleation activity of centrobin in both the centrosome and cytoplasm. Cell Cycle 14 1925-1931. (doi:10.1080/15384101.2015.1041683)

Sironen A, Hansen J, Thomsen B, Andersson M, Vilkki J, Toppari J \& Kotaja N 2010 Expression of SPEF2 during mouse spermatogenesis and identification of IFT20 as an interacting protein. Biology of Reproduction 82 580-590. (doi:10.1095/biolreprod.108.074971)

Sironen A, Kotaja N, Mulhern H, Wyatt TA, Sisson JH, Pavlik JA, Miiluniemi M, Fleming MD \& Lee L 2011 Loss of SPEF2 function in mice results in spermatogenesis defects and primary ciliary dyskinesia. Biology of Reproduction 85 690-701. (doi:10.1095/biolreprod.111. 091132)

Sun X, Kovacs T, Hu YJ \& Yang WX 2011 The role of actin and myosin during spermatogenesis. Molecular Biology Reports 38 3993-4001. (doi:10.1007/s11033-010-0517-0)
Suzuki-Toyota F, Ito C, Toyama Y, Maekawa M, Yao R, Noda T \& Toshimori K 2004 The coiled tail of the round-headed spermatozoa appears during epididymal passage in GOPC-deficient mice. Archives of Histology and Cytology 67 361-371. (doi:10.1679/aohc.67.361)

Suzuki-Toyota F, Ito C, Toyama Y, Maekawa M, Yao R, Noda T, lida H \& Toshimori K 2007 Factors maintaining normal sperm tail structure during epididymal maturation studied in Gopc ${ }^{-1-}$ mice. Biology of Reproduction 77 71-82. (doi:10.1095/biolreprod.106.058735)

Tanaka H, Iguchi N, Isotani A, Kitamura K, Toyama Y, Matsuoka Y, Onishi M, Masai K, Maekawa M, Toshimori K, Okabe $M$ \& Nishimune $Y$ 2005 HANP1/H1T2, a novel histone $\mathrm{H} 1$-like protein involved in nuclear formation and sperm fertility. Molecular and Cellular Biology 25 7107-7119. (doi:10.1128/MCB.25.16.7107-7119.2005)

Tarsounas M, Pearlman RE \& Moens PB 2001 CLIP-50 immunolocalization during mouse spermiogenesis suggests a role in shaping the sperm nucleus. Developmental Biology 236 400-410. (doi:10.1006/dbio. 2001.0286)

Toba S, Koyasako K, Yasunaga T \& Hirotsune S 2015 Lis1 restricts the conformational changes in cytoplasmic dynein on microtubules. Microscopy 64 419-427. (doi:10.1093/jmicro/dfv055)

Wang W, Zhu JQ, Yu HM, Tan FQ \& Yang WX 2010 KIFC1-like motor protein associates with the cephalopod manchette and participates in sperm nuclear morphogenesis in Octopus tankahkeei. PloS ONE 5 e15616. (doi:10.1371/journal.pone.0015616)

Wang S, Ketcham SA, Schon A, Goodman B, Wang Y, Yates JII, Freire E, Schroer TA \& Zheng Y 2013 Nudel/NudE and Lis1 promote dynein and dynactin interaction in the context of spindle morphogenesis. Molecular Biology of the Cell 24 3522-3533. (doi:10.1091/mbc.E1305-0283)

Xu X, Toselli PA, Russell LD \& Seldin DC 1999 Globozoospermia in mice lacking the casein kinase II $\alpha^{\prime}$ catalytic subunit. Nature Genetics 23 118-121. (doi:10.1038/12729)

Yang WX, Jefferson H \& Sperry AO 2006 The molecular motor KIFC1 associates with a complex containing nucleoporin NUP62 that is regulated during development and by the small GTPase RAN. Biology of Reproduction 74 684-690. (doi:10.1095/biolreprod.105.049312)

Yoshida T, Ioshii SO, Imanaka-Yoshida K \& Izutsu K 1994 Association of cytoplasmic dynein with manchette microtubules and spermatid nuclear envelope during spermiogenesis in rats. Journal of Cell Science $\mathbf{1 0 7}$ $625-633$.

Zhang Z, Kostetskii I, Moss SB, Jones BH, Ho C, Wang H, Kishida T, Gerton GL, Radice GL \& Strauss JFIII 2004 Haploinsufficiency for the murine orthologue of Chlamydomonas PF20 disrupts spermatogenesis. PNAS 101 12946-12951. (doi:10.1073/pnas.0404280101)

Zhang Z, Kostetskii I, Tang W, Haig-Ladewig L, Sapiro R, Wei Z, Patel AM, Bennett J, Gerton GL, Moss SB, Radice GL \& Strauss JFIII 2006 Deficiency of SPAG16L causes male infertility associated with impaired sperm motility. Biology of Reproduction 74 751-759. (doi:10.1095/ biolreprod.105.049254)

Zheng H, Stratton CJ, Morozumi K, Jin J, Yanagimachi R \& Yan W 2007 Lack of Spem1 causes aberrant cytoplasm removal, sperm deformation, and male infertility. PNAS 104 6852-6857. (doi:10.1073/pnas. 0701669104)

Zhou J, Du YR, Qin WH, Hu YG, Huang YN, Bao L, Han D, Mansouri A \& Xu GL 2009 RIM-BP3 is a manchette-associated protein essential for spermiogenesis. Development 136 373-382. (doi:10.1242/dev.030858)

Zhou J, Yang F, Leu NA \& Wang PJ 2012 MNS1 is essential for spermiogenesis and motile ciliary functions in mice. PLoS Genetics 8 e1002516. (doi:10.1371/journal.pgen.1002516)

Received 4 July 2015

First decision 6 August 2015

Revised manuscript received 15 January 2016

Accepted 20 January 2016 